Diclofenac (RR 3,98) führend. Außer Naproxen waren aber auch alle anderen Substanzen mit einem erhöhten Risiko assoziiert.

Der kombinierte Endpunkt aus Herzinfarkt, Schlaganfall oder Tod aus kardiovaskulärer Ursache trat unter allen NSAR häufiger auf als unter Placebo. Unter Naproxen war der Risikoanstieg allerdings nicht signifikant.

\section{„Reale“" Patienten stärker gefährdet}

Da die absoluten Fallzahlen in den Studien klein waren, sind die Rate Ratios mit einer gewissen Ungenauigkeit behaftet, wie Jüni einräumt. Trotz dieser Unsicherheit gebe es aber „kaum Belege dafür, dass eines der untersuchten NSAR in kardiovaskulärer Hinsicht sicher sei“.

Aus den geringen Ereigniszahlen dürfe auch nicht auf mangelnde klinische Relevanz geschlossen werden: „Unter Alltagsbedingungen sind die ,Numbers Needed to Harm' niedriger", so Jüni. Anders als die Studienteilnehmer haben die meisten NSAR-Anwender in der Praxis nämlich ohnehin ein mittleres bis hohes Risiko für kardiovaskuläre Ereignisse.

Die Studie bestätigt, dass die COX2-Selektivität für das kardiovaskuläre Risiko einer NSAR-Therapie kein Maß darstellt. Daher kommt die Verschiebung des Gleichgewichts zwischen Prostazyklin und Thromboxan A2 nicht als alleinige Ursache für den Risikoanstieg infrage.

Effekte auf das Gefäßendothel und die NO-Produktion sowie der Blutdruckanstieg und die Volumenretention tragen vermutlich auch dazu bei.

\section{Alternativen zur NSAR-Therapie untersuchen}

„Wenn man ein NSAR verordnet - egal welches -, muss man das kardiovaskuläre Risiko berücksichtigen“, betont Jüni. Generell scheint Naproxen das in kardiovaskulärer Hinsicht unbedenk- lichste Analgetikum für Arthrosepatienten zu sein. Allerdings muss dieser Vorteil gegen die gastrointestinale Toxizität und die notwendige Begleittherapie mit einem Protonenpumpenhemmer abgewogen werden.

„NSAR sind im Hinblick auf Wirkung und Sicherheit keine idealen Medikamente“, heißt es in einem BMJKommentar zu der Studie. Es sei mög- lichweiser an der Zeit, Alternativen zur NSAR-Therapie wie Paracetamol und Opioide systematischer zu untersuchen.

(Beate Schumacher)

Trelle $S$ et al. Cardiovascularr safety of nonsteroidal anti-inflammatory drugs: network meta-analysis. BMJ. 2011; 342:c7086.doi: 10.1136/bmj.c7086

Ray WA. Cardiovas cular Safety of NSAIDs. BMJ. 2011;342:c6618.doi: 10.1136/bmj.c6618

\title{
Wie halten es Fachärzte mit der NSAR-Gabe? Das sagt der Orthopäde:
}

\section{Welche Konsequenzen hat der kardio- vaskuläre Risikoanstieg für die Lang- zeittherapie mit NSAR?}

Aufgrund des kardiovaskulären Risikos sollten bestimmte Patienten von einer NSAR-Therapie ausgenommen werden. Das sind Patienten mit einer Herzinsuffizienz der NYHA-Stadien II-IV, einer arteriellen Hypertonie mit Werten, die anhaltend über 140/90 mmHg liegen, einer klinisch gesicherten KHK, pAVK oder einer zerebrovaskulären Erkrankung. Weitere Kontraindikationen sind natürlich ein peptisches Ulkus oder eine aktive gastrointestinale Blutung, entzündliche Darmerkrankungen, schwere Leberinsuffizienz, Niereninsuffizienz $\mathrm{ab}$ einer Kreatinin-Clearance unter $30 \mathrm{ml} / \mathrm{min}$ sowie Schwangerschaft und Stillzeit. Aufgrund des günstigeren Nebenwirkungsprofils hinsichtlich gastrointestinaler Komplikationen setzen wir in der Langzeittherapie bevorzugt selektive COX-2-Hemmer ein.

Wirkt sich der Anstieg des Herz-Kreislauf-Risikos auch auf die Kurzzeittherapie mit NSAR aus?

Nicht bei kardiovaskulär unauffälligen Patienten. Bei Patienten mit kardiovaskulären Vorerkrankungen sollte das Nutzen-Risiko-Verhältnis besonders sorgfältig abgewogen werden. Im Falle der Entscheidung für eine The-

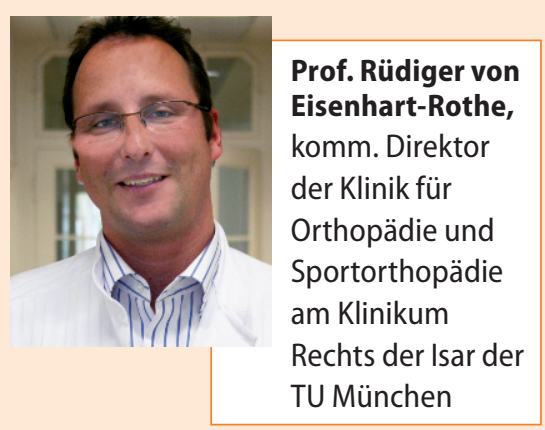

rapie sollten die niedrigste wirksame Dosis und eine möglichst kurze Therapiedauer gewählt werden. Patienten mit Herzinsuffizienz der NYHA-Stadien II-IV, nicht kontrolliertem Bluthochdruck, symptomatischer KHK, pAVK oder zerebrovaskulären Erkrankungen sollten auch nicht kurzfristig mit NSAR behandelt werden.

Welche medikamentösen Alternativen zu NSAR gibt es für die analgetische Behandlung?

Speziell bei Patienten mit leichter Arthrose kommen DMOAD (disease modifying osteoarthritis drugs) infrage, also die intraartikuläre Injektion von Hyaluronsäure oder die Einnahme von Glucosamin oder Chondroitin. Beide Therapien sind in den Behandlungsleitlinien der OARSI (Osteoarthritis Research Society International) bei milden Arthrosegraden mit dem Evidenzlevel Ia bewertet. 